\title{
BJMHR
}

British Journal of Medical and Health Research

Journal home page: www.bjmhr.com

\section{Organophosphorous Poisoning Induced Parkinsonism - An Uncommon Manifestation of A Common Poison \\ Padma Deepika $\mathbf{M}^{*}$, Sunil Kumar, Jay Patel \\ Department of General Medicine, SBKS MIRC, Sumandeep Vidyapeeth, Waghodia, Piparia, Vadodara - 391760}

\section{ABSTRACT}

Organophosphate insecticide (OPI) poisoning is one of the most common poisonings seen in developing countries. The usual neurological complications of OPI compounds are acute cholinergic crisis, intermediate syndrome, and delayed sensorimotor polyneuropathy. Extrapyramidal symptoms, though very rare, can also occur following OPI and pyrethroid exposure. Parkinsonism is a primary neurotoxic manifestation of organophosphate pesticide intoxication. In addition, a limited number of case studies are available describing extrapyramidal manifestations after acute organophosphate poisoning such as dystonias, rest tremors, cog wheel rigidity, and choreoathetosis. The development of typical parkinsonism following brief inhalational of chlorpyrifos (Organophosphorous compound) and cypermethrin exposure is very rare. We report a 36-year-old female who developed parkinsonism following acute accidental inhalational exposure to, Cypermethrin 5\% and chlorpyriphos 50\% (an OPI compound). She developed features of parkinsonism on day 8 of hospital admission, but recovered gradually in 15 days and had a marked improvement after treatment with amantadine.

Keywords: Acetyl cholinesterase, cogwheel rigidity, cypermethrin, chlorpyriphos, organophosphate insecticide, Parkinsonism. 


\section{INTRODUCTION}

The organophosphate insecticides (OPI) are chemical substances that act by inhibiting the acetylcholinesterase enzyme (AChE) activity. The acute phases of organophosphate poisoning have symptoms which include muscarinic actions such as miosis, hypersalivation, and nicotinic actions such as muscle weakness and fasciculations. After the initial remission of these acute cholinergic crises, there is an intermediate phase of neuropathy and muscle weakness (intermediate syndrome). Delayed polyneuropathies emerge later, and finally a phase involving the appearance of neuropsychiatric manifestations is possible. But very rarely it may produce extrapyramidal symptoms. Cypermethrin is a class II synthetic pyrethroid pesticide and it is one of the most widely used insecticides. It crosses the blood brain barrier and exerts neurotoxicity in the central nervous system. Cypermethrin-mediated neurotoxicity is contributed by its ability to induce free radical generation, inhibition of antioxidant defense system and inhibition of mitochondrial electron transport chain. Since oxidative stress critically contributes to the nigrostriatal dopaminergic neurodegeneration, cypermethrin could be considered as one of the most relevant pesticides, which possibly implicates in Parkinson's disease pathogenesis.

\section{CASE REPORT}

A 36 year old female patient presented to our emergency department with history of headache, giddiness and nausea following brief accidental inhalation exposure of an organophosphate insecticide (OPI) compound, (cypermethrin 5\% and chlorpyrifos 50\%). She was treated with atropine, pralidoxime, $2 \mathrm{gm}$ iv bolus followed by $500 \mathrm{mg} / \mathrm{hr}$ infusion for $48 \mathrm{hrs}$.

On examination, she was conscious but disoriented. Vitals were stable. Pupils were bilaterally pin point. Systemic examination was normal. Her biochemical values were normal including arterial blood gas analysis. Her ECG, Chest X-ray were normal except serum cholinesterase level which was very low (2190 IU/L) normal value is $4000 \mathrm{IU} / \mathrm{L}$ to $11000 \mathrm{IU} / \mathrm{L}$.

From the day 8 onward, the patient rapidly developed extrapyramidal manifestations. There was rigidity and bradykinesia, mask like face with decreased blinking rate, slow saccades, broken pursuits, monotonous speech, rest tremors, and cogwheel rigidity. The deep tendon reflexes were brisk in both upper limb and left knee and ankle jerk was absent, power of the all four limbs was grade 3/5, with bilateral mute plantar response, and fasciculations were present. Pupils were persistantly pin point. MRI brain was done which showed hyperintense signal in bilateral caudate nucleus, putamen and pulvinar of thalamus in T2 and FLAIR images. CSF examination showed 2 cells, all lymphocytes, Protein was $392 \mathrm{mg} / \mathrm{dl}$, sugar $84 \mathrm{mg} / \mathrm{dl}$ against a corresponding blood sugar of $103 \mathrm{mg} / \mathrm{dl}$. CSF IgM for Japanese Encephalitis was negative. A slit lamp examination for Kayser Fleischer ring was negative. EEG showed 
generalized slowing and there is no interictal or periodic discharge. She was treated with Tab. Amantadine $100 \mathrm{mg}$ bd and trihexyphenidyl $2 \mathrm{mg}$ tds. Her condition gradually improved. Follow up MRI brain done after 6 months did not show any abnormality.

\section{DISCUSSION}

In Organophosphate insecticide (OPI) poison, there is spectrum of delayed neurological manifestations like Intermediate syndrome, Organophosphrous induced neuropsychiatric disturbances1. Each one has specific features and period of occurrence from ingestion of Organophosphate Compound (OPC). Intermediate syndrome (IMS) presents in 1-4 days after ingestion with weakness of proximal muscles, neck muscles, extraocular muscles and involvement of cranial nerves. Organophosphrous induced neuropsychiatric disturbance (OPIND), manifests 2-3 weeks after ingestion of OPC as weakness of distal muscles, with or without sensory involvement.

Only $0.5 \%$ of organophosphate poisoning patients develop neurotoxic manifestations in the form of extrapyramidal syndromes such as parkinsonism. ${ }^{2}$ The first report of parkinsonism following OPI exposure was in 1978 by Davis et $a l^{3}$. In 1999 Bhatt et al. reported five patients with OPI-induced parkinsonism, among whom four cases occurred following inhalational exposure to OPI. The phosphate-containing OPI compounds such as chlorpyriphos appear to exhibit more neurotoxicity than other compounds ${ }^{7}$. The exact latent period before onset of parkinsonism following OPI exposure is uncertain and varies in different reports. After an acute cholinergic crisis, patients may develop parkinsonism symptoms including rigidity, tremors, bradykinesia, and masked face. Eighty-one per cent of patients with extrapyramidal syndrome developed the symptoms within 2 weeks of their poisoning ${ }^{2,4}$. Seventy-seven per cent of patients with extrapyramidal symptoms recovered, and $71 \%$ of the patients recovered without antiparkinsonian drug treatments ${ }^{2,4}$. The duration of extrapyramidal symptoms ranges from 7 days to 2 months 2 . The mechanisms of extrapyramidal parkinsonism, however, remain unclear $^{2,6}$. The excessive acetylcholine activity due to prolonged and irreversible inhibition of acetylcholinesterase during OPI poisoning may alter the dopamine activity within the basal ganglia and substantia nigra, resulting in the exposed person exhibiting parkinsonism-like features. ${ }^{2,5}$

One study was undertaken to investigate the effects of cypermethrin on Dopamine transporter (DAT), vesicular monoamine transporter 2 (VMAT 2). Dopamine transporter (DAT) and vesicular monoamine transporter 2 (VMAT 2) regulate dopamine level in the nigrostriatal dopaminergic neurons. DAT mediates the removal of dopamine from the synapse to the intracellular space for recycling and metabolism. VMAT 2, on the other hand, transports cytoplasmic dopamine into vesicles for storage and release and protects it from oxidation. DAT 
or VMAT 2 dysfunctions or their abnormal expressions lead to dopamine oxidation, thereby free radical generation, one of the major causes of the nigrostriatal neurodegeneration ${ }^{5,7}$. Higher ratio of DAT/VMAT 2 is observed in the terminals of dopaminergic neurons in pesticide-induced PD phenotype ${ }^{7}$. The decreased VMAT 2 level directly reflects loss of the ability of dopaminergic neurons to protect against cypermethrin-induced neurotoxicity after prolonged exposure, as VMAT 2 is well known to protect dopaminergic neurons through vesicular sequestration of toxic metabolites ${ }^{7}$. The reduced VMAT 2 is not an unusual phenomenon, as it is involved in the transportation of dopamine into vesicles for storage, release and to protect from autooxidation. The reduced VMAT 2 could also be explained by the fact that cypermethrin reduced the level of dopamine ${ }^{7}$.

The imaging findings in OPI-induced parkinsonism vary from normal appearance to symmetric signal changes in the basal ganglia. However SPECT imaging in patient of parkinsonism due to organophosphrous poisoning showing hyperperfusion of putamen contributes to the manifestation of parkinsonism in victims of organophosphate poisoning ${ }^{8}$. OPI-induced acute parkinsonism is a reversible phenomenon. There are reports of recovery from OPI-induced parkinsonism without any treatment. Treatment can be undertaken if the symptoms are prolonged and distressing. The selection of the suitable drug for the management of OPIinduced parkinsonism is also a challenging task. The drugs reported to be effective in case reports of OPI-induced parkinsonism are bromocriptine, benzhexol, amantadine, and biperidine. Levodopa was not effective in the treatment of OPI-induced Parkinsonism.
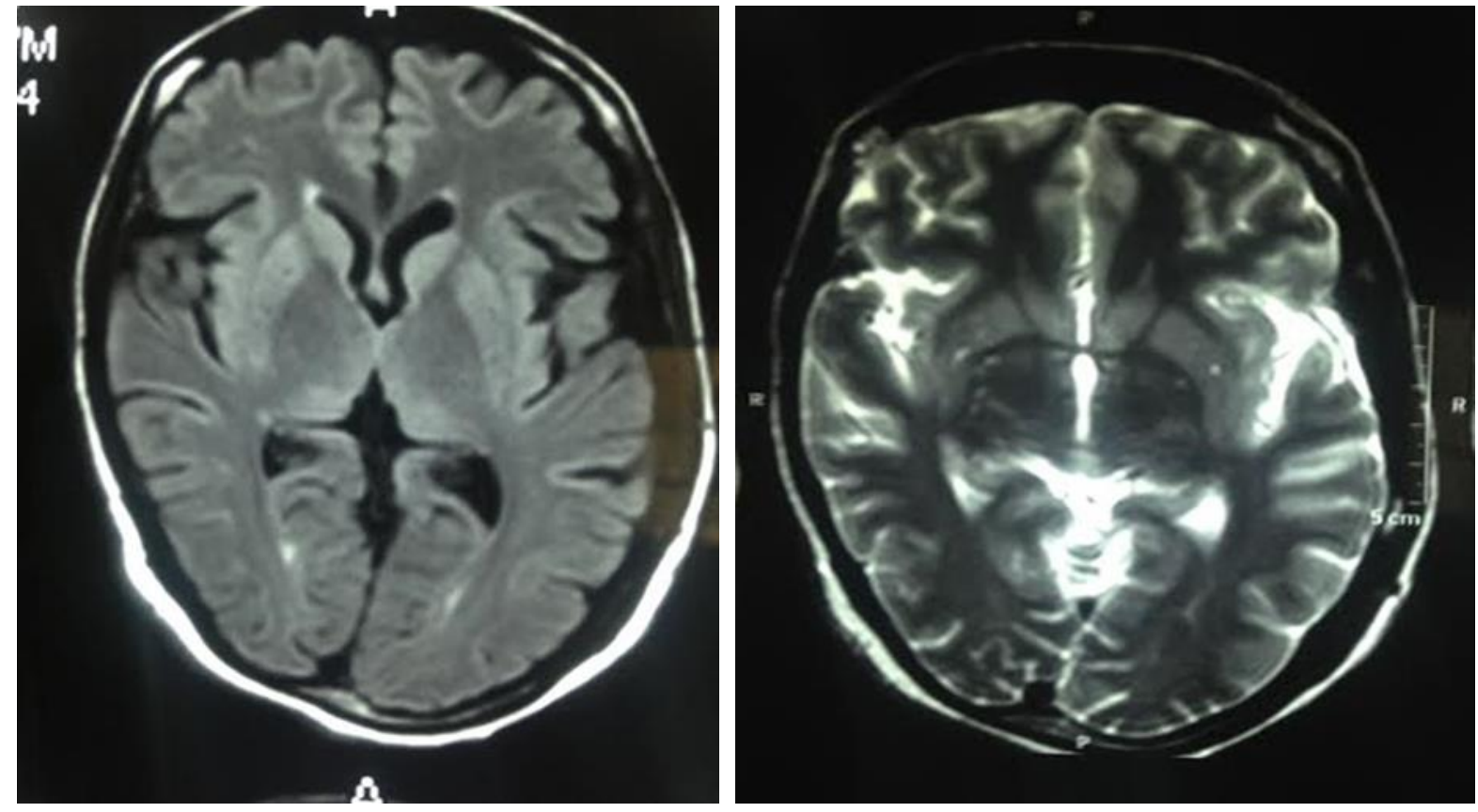

Figure 1a and 1b: MRI Brain:-T2 \& FLAIR images showing a hyper intensity signal in bilateral caudate nucleus, putamen and pulvinar of thalamus. 


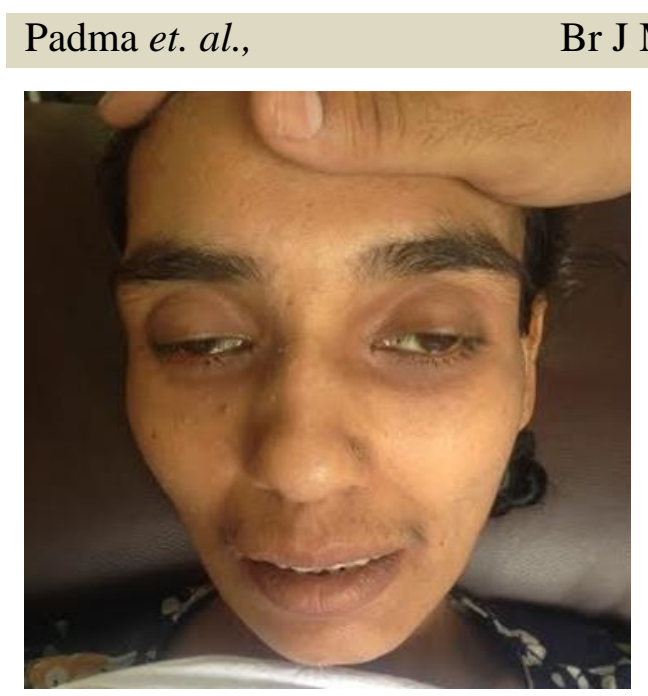

ISSN: 2394-2967
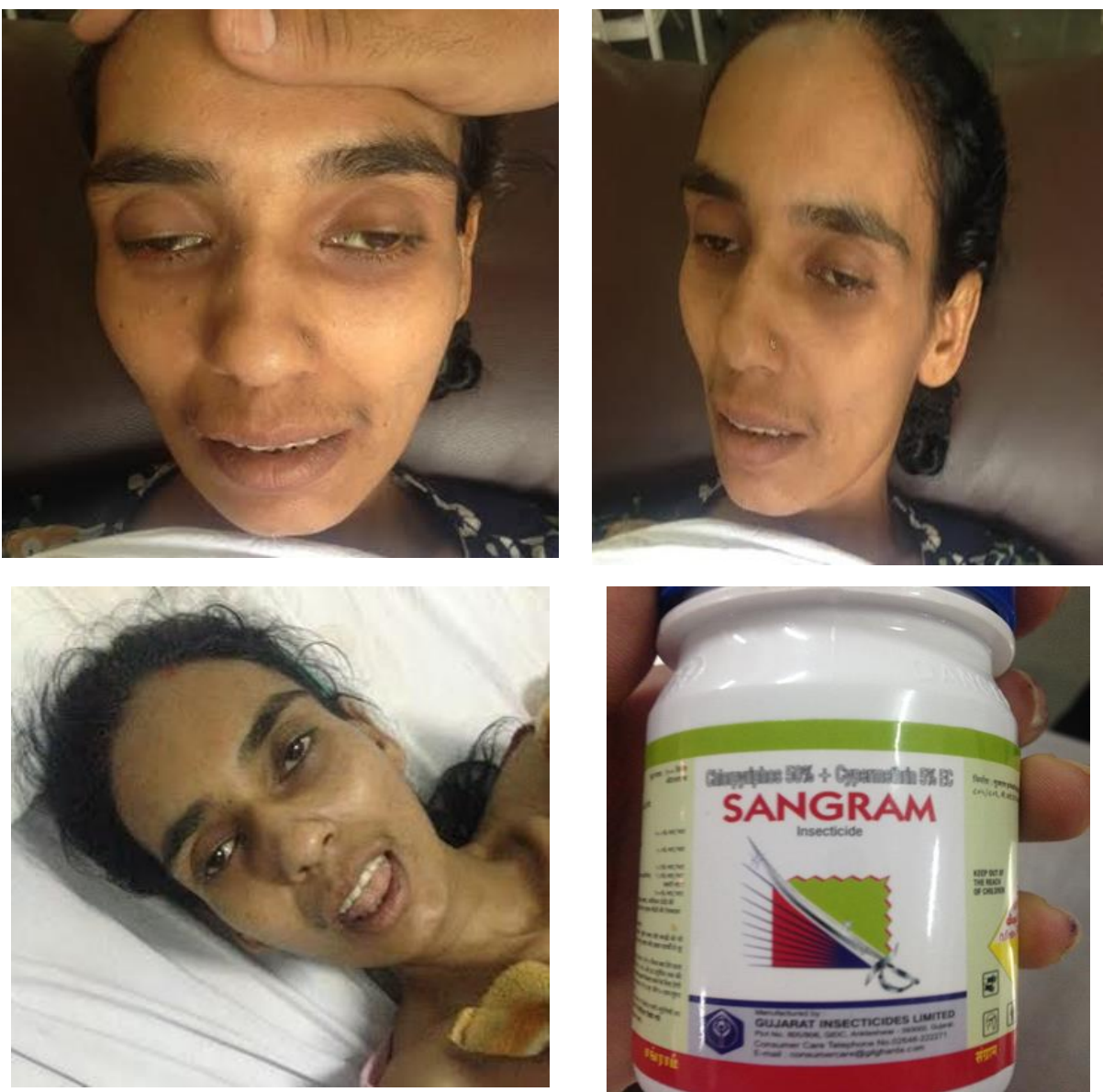

Figure 2a, 2b \&2c showing mask like face.

\section{REFERENCES:}

1. Bhatt MH, Elias MA, Mankodi AK. Acute and reversible Parkinsonism due to organophosphate pesticide intoxication: Five cases. Neurology 1999; 52:1467-71.

2. Hsieh BH, Deng JF, Ger J, Tsai WJ. Acetylcholinesterase Inhibition and the Extrapyramidal Syndrome: A Review of the Neurotoxicity of Organophosphate. Neuro Toxicology 2001;22:423-7

3. Kiyotaka Nakamagoel, Masahiko Watanabe, Tohoru Takeda, Taro Mizutani, Akira Tamaoka. Parkinsonism with organophosphate poisoning BMJ Case Reports 2009;doi:10.1136/bcr.04.2009.1766.

4. Shahar E, Andraws J. Extra-pyramidal Parkinsonism complicating organophosphate insecticide poisoning. Eur J Paediatr Neurol 2001;5:261-4.

5. Goel D, Singhal A, Srivastav RK, Verma A, Lamba A. Magnetic resonance imaging changes in a case of extra-pyramidal syndrome after acute organophosphate poisoning. Neurol India 2006;54:207-9.

6. Huaijun Liu, Yanmei Yang, Jiping Yang, et al Brain injury due to acute organo phosphate poisoning : magnetic resonance imaging manifestation and pathological characteristics. Nerve Regeneration Research 2007,2:403. 
7. Eddleston M, Buckley NA, Eyer P, Dawson AH. Management of acute organophosphorus pesticide poisoning. Lancet 2008;371:597-607.

8. Tanima Das, Indira Maisnam, Arup K Kundu, SP Saha, Sandip Ghosh, Anupam Maity Atypical Central Nervous System Involvement in Acute Organophosphorous Poisoning.JAPI may 2011

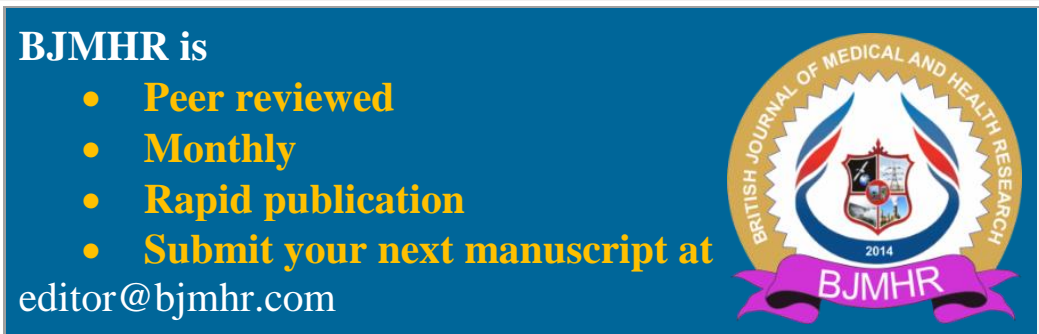

